\title{
Magnetic field influence on the proximity effect in semiconductor-superconductor hybrid structures and their thermal conductance
}

\author{
Grygoriy Tkachov ${ }^{1,2}$ and Vladimir I. Fal'ko ${ }^{1}$ \\ ${ }^{1}$ Department of Physics, Lancaster University, Lancaster LA1 4YB, United Kingdom \\ ${ }^{2}$ Institute for Radiophysics and Electronics NAS, Kharkiv 61085, Ukraine
}

(Received 3 July 2003; revised manuscript received 13 November 2003; published 10 March 2004)

\begin{abstract}
We show that a magnetic field can influence the proximity effect in NS junctions via diamagnetic screening current flowing in the superconductor. Using ballistic quasi-one-dimensional (Q1D) electron channels as an example, we show that the supercurrent flow shifts the proximity-induced minigap in the excitation spectrum of a Q1D system from the Fermi level to higher quasiparticle energies. Thermal conductance of a Q1D channel (normalized by that of a normal Q1D ballistic system) is predicted to manifest such a spectral feature as a nonmonotonic behavior at temperatures corresponding to the energy of excitation into the gapful part of the spectrum.
\end{abstract}

DOI: 10.1103/PhysRevB.69.092503

PACS number(s): 74.45.+c, 74.50.+r, 73.23.Ad

The superconducting proximity effect is a mesoscopic scale phenomenon, which consists of the penetration and coherent propagation of Cooper pairs from a superconductor $(\mathrm{S})$ into a normal metal $(\mathrm{N})$. The Cooper pair transfer into the normal metal can be equivalently described as an Andreev reflection process ${ }^{1}$ which consists of electron (with momentum p) conversion into the Fermi sea hole (with momentum - p) at the NS interface. The interference between an electron and the Andreev reflected hole imposes a minigap onto the spectrum of quasiparticle excitation near the Fermi level in the normal part of such a hybrid structure, ${ }^{2}$ thus giving rise to pronounced features in its $I(V)$ characteristics $^{3-5}$ and thermoelectric properties. ${ }^{6}$ Studies of the proximity effect have recently been made in various combinations of materials, including junctions between superconductors and semiconductor structures ${ }^{3}$ supporting a two-dimensional electron gas. In the case of electrons in a semiconductor structure weakly coupled to a superconductor, the minigap value discussed in the literature 7,8 is much smaller than that of the "mother" gap in the superconductor, both due to the mismatch $v_{F} \ll v_{S}$ between Fermi velocities in the twodimensional gas $\left[v_{F}=\left(2 E_{F} / m\right)^{1 / 2}\right]$ and a superconducting metal $\left(v_{S}\right)$, and also due to a possible Schottky barrier between them, with transparency $\theta \sim e^{-2 a / \lambda}$ (dependent on the length $\lambda$ of electron penetration into the barrier of the thickness $a), E_{g} \approx\left(v_{F} / v_{S}\right) \theta E_{F} \ll \Delta$.

It has been noticed that the electron-hole interferences and the SN proximity effect in hybride structures survive at higher magnetic fields than the weak localization-another quantum interference effect. ${ }^{3-5}$ This has been understood as a consequence of the fact that the interfering electron and Andreev-reflected hole retrace the same geometrical path in the normal metal, thus hardly encircling any magnetic flux. ${ }^{9}$ Therefore, another mechanism of magnetic field influence on the superconducting proximity needs to be taken into account, via a screening diamagnetic supercurrent on the S-side of the hybrid structure. Since Andreev reflection takes place at the NS interface, where Cooper pairs flow, the incoming electron and the hole reflected by a moving condensate of Cooper pairs would be slightly shifted in momentum space; hence the ideal condition for them to retrace the same geometrical path is violated. As the orbital effect of the magnetic field on the normal metal or semiconductor side of the system is weak, the influence via diamagnetic screening may be the major factor of magnetic field influence on the superconducting proximity effect.

Below, we analyze the influence of diamagnetic supercurrent in the system where the latter would be the only way a magnetic field might affect the proximity effect: a ballistic one-dimensional conductor connected in parallel to a superconducting bulk [Fig. 1(a)]. To be specific, we model such a conductor as a quasi-one-dimensional (Q1D) channel formed near the edge of a 2D electron gas in a heterostructure $(x-y$ plane) with the side contact to a superconducting film, by depleting the 2D gas using a split top gate, and subjected to a weak magnetic field $\mathbf{B}=(0 ; 0 ; B)$. We show that the spectrum of low-energy quasiparticle excitiation in such a hybrid system has the minigap displaced with respect to the Fermi level to higher energies,

$$
\epsilon_{\alpha p}^{ \pm}=v_{F} \Pi \times \operatorname{sgn} p-\alpha \varepsilon_{Z} \pm \sqrt{v_{F}^{2}\left(|p|-p_{F}\right)^{2}+E_{g}^{2}},
$$

reflecting the fact that Cooper pairs in the channel are forced into the flow while tunneling from the bulk of the superconductor (where they are formed of two electrons with exactly opposite momenta) across the region of penetration of the magnetic field. [The Zeeman splitting effect is also taken care of by the term $\alpha \varepsilon_{Z}$ ( $\alpha$ is the spin projection) in Eq. (1)]. As a result, each of the two electrons acquires the momentum shift

$$
\Pi=\frac{e B \delta}{c} \tanh \frac{L}{2 \delta}
$$

caused by the Lorentz force and equal to the difference between the vector potential $\mathbf{A}=(0, A, 0)$ deep inside the superconductor, $A=0$, and, at its surface, $A=B \delta \tanh (L / 2 \delta)$, where $\delta$ and $L$ stand for the London penetration depth and the superconductor film thickness, respectively. The spectrum described by Eq. (1) can also be understood as one of the Bogolubov quasiparticles in the laboratory frame, where 


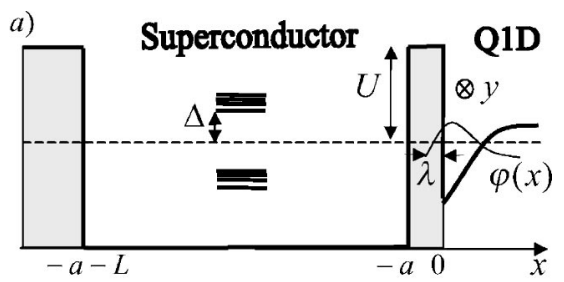

b)

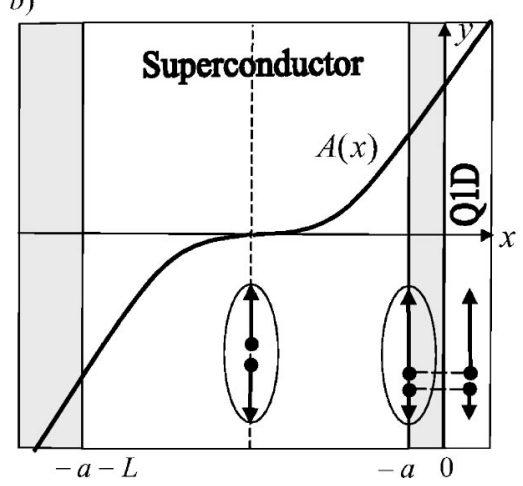

FIG. 1. (a) Schematic view of a superconductor/Q1D system junction. (b) Vector potential profile.

the equilibrium conditions are set by the heat reservoirs, for the condensate moving along the Q1D channel with the drift velocity $\Pi / m$ ( $m$ is the effective electron in the semiconductor). According to Eq. (1) the minigap is removed from the Fermi level when the field reaches the value

$$
B^{*} \approx B_{c 1} \frac{\delta}{\xi} \operatorname{coth}\left(\frac{L}{2 \delta}\right) \frac{\theta E_{F}}{\Delta} \ll B_{c 1},
$$

where $B_{c 1}$ and $\xi$ are the first critical field and the coherence length in the superconductor.

The removal of a minigap from the Fermi level caused by a magnetic field would manifest itself in the transport properties of a hybrid sytem, such as the electron-mediated heat transfer. The ballistic quasiparticle spectrum in Eq. (1) gives rise to the thermal conductance

$$
\kappa(T, B)=\kappa_{N}(T) \times \frac{3}{4 \pi^{2}} \sum_{ \pm} \int_{\left(E_{g} \pm v_{F} \Pi\right) / k_{B} T}^{\infty} \frac{x^{2} d x}{\cosh ^{2} \frac{x}{2}}
$$

where $\kappa_{N}(T)=\pi k_{B}^{2} T / 3 \hbar$ is the conductance of a normal quantum ballistic wire. ${ }^{10}$ At a zero magnetic field, the temperature dependence of $\kappa$ is activational, $\kappa\left(T<E_{g} / k_{B}\right)$ $\propto e^{-E_{g} / k_{B} T}$, whereas at high fields, when there is no gap at the Fermi energy, $\kappa(T, B)=\kappa_{N}(T)$. The crossover from low to high fields takes place at $B^{*}[$ Eq. (3)] and reflects the presence of a minigap $E_{g}$ in the quasiparticle spectrum at finite excitation energies. This results in a nonmonotonic temperature and magnetic field dependence of the ratio $\kappa(T, B) / \kappa_{N}(T)$.

The analysis of the quasiparticle spectrum formed due to multiple Andreev reflections in this paper is based on the standard weak-coupling approach to the proximity effect description in superconductor junctions with normal metals and electron layers in semiconductors. ${ }^{7}$ To be specific, we describe the Q1D confinement (provided by a gate) by the $2 \mathrm{D}$ electron wave function $\varphi(x)$ localized in the $x$ direction, whose magnitude at the interface can be estimated from the boundary condition $\varphi(0)=\lambda \partial_{x} \varphi(0)$, with $\lambda$ standing for the electron penetration length into the barrier. The Fermi momentum of the Q1D system $p_{F}$ and 3D electron density on the semiconductor side are assumed to be much smaller than those in the superconductor, and we also take the tunneling coefficient $\theta \sim \exp (-2 a / \lambda)$ as a small parameter. These assumptions enable us to neglect the influence of the normal system on the superconductor and to investigate the proximity effect in the Q1D system without feedback.

In the presence of a magnetic field $\mathbf{B}=(0 ; 0 ; B)$ it is convenient to choose the vector potential to be parallel to the interface, $\mathbf{A}(x)=[0, A(x), 0]$ in order to deal with a real order parameter in the superconductor. The vector potential $A(x)$ acting on the normal electrons must be found selfconsistently, taking into account the screening of the external magnetic field $B$ by a diamagnetic supercurrent. ${ }^{2,11}$ Inside the superconductor $A(x)$ can be found from the London equation with the boundary conditions $\partial_{x} A(-a)=B$ and $\partial_{x} A(-a$ $-L)=B$ as follows:

$$
A(x)=B \delta \frac{\sinh [(x+a+L / 2) / \delta]}{\cosh (L / 2 \delta)} .
$$

It is antisymmetric with respect to the middle of the superconductor: $A(x=-a-L / 2)=0[$ Fig. 1(b)]. Since $A(x)$ must be continuous at the surface of the superconductor $x=-a$, in the semiconductor $x \geqslant-a$ it varies as $A(x)=B(x+a)$ $+B \delta \tanh (L / 2 \delta)$. The width of the electronic wave function in the Q1D channel, $\delta x \sim k_{F}^{-1}$ and the barrier thickness $a$ are both much less than $L$ or $\delta$; therefore, the vector potential acting on the Q1D electrons is virtually a constant: $A(x)$ $\approx A(-a)=B \delta \tanh (L / 2 \delta)$, which will be used below to determine the quasiparticle spectrum in the channel.

We describe superconducting correlations in the Q1D channel using a pair of coupled equations for $\hat{\psi}_{p}(t)$ $=\left(\begin{array}{c}\psi_{\alpha p}(t) \\ \psi_{-\alpha p}(t)\end{array}\right)$ and $\hat{\psi}_{p}^{\dagger}(t)=\left(\begin{array}{c}\psi_{\alpha p}^{\dagger}(t) \\ \psi_{-\alpha p}^{\dagger}(t)\end{array}\right)$-creation and annihilation operators:

$$
\begin{aligned}
& {\left[i \hbar \partial_{t}-\frac{(p+\Pi)^{2}}{2 m}+\sigma_{3} \varepsilon_{Z}+E_{F}\right] \hat{\psi}_{p}(t)} \\
& \quad=\vartheta^{1 / 2} \int d t^{\prime}\left[G\left(t, t^{\prime}\right) \hat{\psi}_{p}\left(t^{\prime}\right)+F^{*}\left(t, t^{\prime}\right) i \sigma_{2} \hat{\psi}_{-p}^{\dagger}\left(t^{\prime}\right)\right], \\
& {\left[-i \hbar \partial_{t}-\frac{(-p+\Pi)^{2}}{2 m}+\sigma_{3} \varepsilon_{Z}+E_{F}\right] \hat{\psi}_{-p}^{\dagger}(t)} \\
& \quad=\vartheta^{1 / 2} \int d t^{\prime}\left[F\left(t, t^{\prime}\right) i \sigma_{2}^{t} \psi_{p}\left(t^{\prime}\right)+G^{*}\left(t, t^{\prime}\right) \hat{\psi}_{-p}^{\dagger}\left(t^{\prime}\right)\right],
\end{aligned}
$$

where 


$$
\vartheta=\left(\frac{\hbar^{2} \varphi(0)}{m_{B} \lambda \exp (a / \lambda)}\right)^{2} \sim\left(\frac{\hbar^{2} k_{F}^{3 / 2}}{m \exp (a / \lambda)}\right)^{2}
$$

characterizes the tunneling coupling to the superconductor and the electron momentum shift in the magnetic field $\Pi$ is related to the vector potential by Eq. (2). In Eq. (5), $G\left(t, t^{\prime}\right) \equiv G\left(x=-a, x^{\prime}=-a, t-t^{\prime}\right) \quad$ and $\quad F\left(t, t^{\prime}\right) \equiv F(x$ $\left.=-a, x^{\prime}=-a, t-t^{\prime}\right)$ are the normal and anomalous Green functions of the superconductor at its boundary; $\sigma_{2}$ and $\sigma_{3}$ are Pauli matrices $\left(\sigma^{t}\right.$ is transposed to $\sigma$ ). Since the size of the Fermi sea in the semiconductor wire is much smaller than in the superconductor, one can ignore the dependence of $G$ and $F$ on the momentum parallel to the interface: only electrons in the superconductor moving nearly perpendicularly to the interface can tunnel into the Q1D wire. Since we are interested in the low-temperature regime $k_{B} T \sim E_{g} \ll \Delta$, we will neglect the terms containing the normal Green function $G$ in Eqs. (5). For the chosen gauge, the anomalous Green function of the superconductor, $F$ in Eqs. (5) has no phase factors, despite the presence of a magnetic field. For a weak field $B \ll B_{c 1}$, its time Fourier transform can be estimated as $F(\epsilon) \approx L^{-1} \Sigma_{p_{x}} \Delta /\left(\Delta^{2}-\epsilon^{2}+\eta_{p_{x}}^{2}\right)$, with $\eta_{p_{x}}$ being the normal electron dispersion near the Fermi level in the superconductor. The integration over the perpendicular momentum $p_{x}$ gives $F(\epsilon) \approx \Delta / \hbar v_{S}\left(\Delta^{2}-\epsilon^{2}\right)^{1 / 2}$, thus giving us the minigap $E_{g}=\vartheta F(\epsilon=0)$ mentioned in the introduction and obtained in earlier publications. ${ }^{7}$

The solution of Eqs. (5) for $\epsilon \ll \Delta$ is given by the Bogolubov transformation of the forms

$$
\begin{aligned}
& \psi_{\alpha p}(t)= u_{p} b_{\alpha p} \exp \left(-i t \epsilon_{\alpha p}^{+} / \hbar\right)+i \sigma_{2}^{\alpha,-\alpha} v_{p} b_{-\alpha-p}^{\dagger} \\
& \times \exp \left(-i t \epsilon_{\alpha p}^{-} / \hbar\right), \\
& u_{p}^{2}=\frac{1}{2}\left[1+\frac{v_{F}\left(|p|-p_{F}\right)}{\left[v_{F}^{2}\left(|p|-p_{F}\right)^{2}+E_{g}^{2}\right]^{1 / 2}}\right], \\
& v_{p}^{2}=1-u_{p}^{2},
\end{aligned}
$$

where $b_{\alpha p}$ and $b_{-\alpha-p}^{\dagger}$ are Bogolubov's quasiparticle operators, and the excitation spectrum $\epsilon_{\alpha p}^{ \pm}$is given by Eq. (1) (see Fig. 2). The Zeeman term in Eq. (1) turns out to be much smaller than the orbital one $E_{Z} / v_{F} \Pi \sim g / k_{F} \min (\delta, L)$ $\ll 1$ - unless the electron g-factor is anomalously large.

Due to the motion of the Q1D condensate the excitation energy curve is tilted by energy $v_{F} \Pi \operatorname{sgn} p$. The field $B^{*}$ [Eq. (3)] at which the minigap is removed from the Fermi level is determined by the condition that $v_{F} \Pi=E_{g}$. Note that at higher fields $B^{*}<B \ll B_{c 1}$, the quasiparticle spectrum remains gapful, with the center of the gap moved to energies $\sim E_{g}$.

Now we turn to the calculation of the thermal conductance $\kappa(T, B)$ of a long Q1D channel whose ends are kept at temperatures $T$ and $T+\Delta T(\Delta T \ll T)$. Since no heat can get into the strongly gaped superconductor, the middle of the wire represents a bottleneck for the heat transport, so that we can analyze $\kappa(T, B)$ in the infinite wire geometry. The expression for the energy current operator $j_{\epsilon}(y t)$ in a wire can

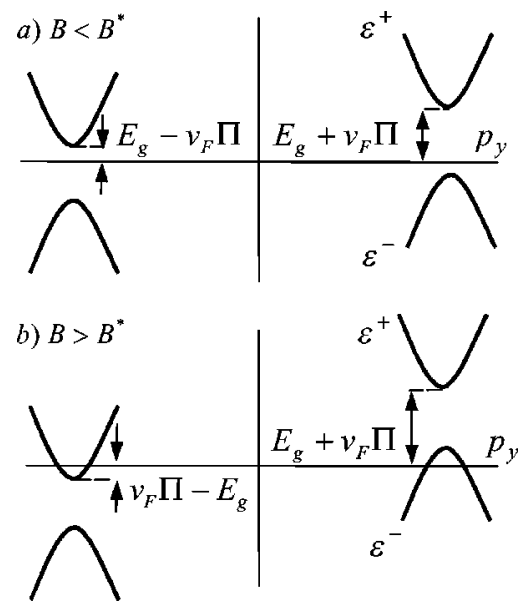

FIG. 2. Schematic view of the quasiparticle spectrum described by Eq. (1).

be found from the continuity equation $\partial_{y} j_{\epsilon}(y t)$ $=-\partial_{t} \rho_{\epsilon}(y t)$, where the density of energy $\rho_{\epsilon}(y t)$ corresponding to the equations of motion (5) is

$$
\begin{aligned}
\rho_{\epsilon}(y t)= & \frac{1}{2} \sum_{\alpha}\left(\psi_{\alpha}^{\dagger}(y t)\left[\frac{(\hat{p}+\Pi)^{2}}{2 m}-\alpha \varepsilon_{Z}-E_{F}\right] \psi_{\alpha}(y t)\right. \\
& \left.-E_{g} i \sigma_{2}^{\alpha,-\alpha} \psi_{-\alpha}^{\dagger}(y t) \psi_{\alpha}^{\dagger}(y t)+\text { H.c. }\right),
\end{aligned}
$$

where $\psi_{\alpha}(y t)=L_{y}^{-1 / 2} \Sigma_{p} \psi_{\alpha}(t) \exp (i p y / \hbar)$ with $L_{y}$ being the length of the Q1D system, and $\hat{p}=-i \hbar \partial_{y}$. Using the Bogo lubov transformation (6) for $\psi_{\alpha}(t)$, for the density of energy one finds

$$
\begin{aligned}
\rho_{\epsilon}(y t)= & \sum_{\alpha p p^{\prime}} \frac{e^{(i y / \hbar)\left(p^{\prime}-p\right)}}{2 L_{y}}\left\{v_{p} v_{p^{\prime}}\left(\epsilon_{\alpha p^{\prime}}^{-}+\epsilon_{\alpha p}^{-}\right)\right. \\
& \times b_{-\alpha-p} b_{-\alpha-p^{\prime}}^{\dagger} e^{-(i t / \hbar)\left(\epsilon_{\alpha p^{\prime}}^{-}-\epsilon_{\alpha p^{-}}^{-}\right)}+u_{p} u_{p^{\prime}} \\
& \times\left(\epsilon_{\alpha p^{\prime}}^{+}+\epsilon_{\alpha p}^{+}\right) b_{\alpha p}^{\dagger} b_{\alpha p^{\prime}} e^{-(i t / \hbar)\left(\epsilon_{\alpha p^{\prime}}^{+}-\epsilon_{\alpha p}^{+}\right)} \\
& +u_{p} v_{p^{\prime}} i \sigma_{2}^{\alpha,-\alpha}\left(\epsilon_{\alpha p}^{+}+\epsilon_{\alpha p^{\prime}}^{-}\right) \\
& \times b_{\alpha p}^{\dagger} b_{-\alpha-p^{\prime}}^{\dagger} e^{(i t / \hbar)\left(\epsilon_{\alpha p}^{+}-\epsilon_{\alpha p^{\prime}}^{-}\right)}+v_{p} u_{p^{\prime}}, i \sigma_{2}^{-\alpha, \alpha} \\
& \left.\times\left(\epsilon_{\alpha p}^{-}+\epsilon_{\alpha p^{\prime}}^{+}\right) b_{-\alpha-p} b_{\alpha p^{\prime}} e^{(i t / \hbar)\left(\epsilon_{\alpha p}^{-}-\epsilon_{\alpha p^{\prime}}^{+}\right)}\right\} .
\end{aligned}
$$

In order to satisfy the continuity equation with $\rho_{\epsilon}(y t)$ given by Eq. (8) the energy current $j_{\epsilon}(y t)$ must have the following form:

$$
\begin{aligned}
j_{\epsilon}(y t)= & \sum_{\alpha p p^{\prime}} \frac{e^{(i y / \hbar)\left(p^{\prime}-p\right)}}{2 L_{y}}\left\{v_{p} v_{p^{\prime}} \frac{\left(\epsilon_{\alpha p^{\prime}}^{-}\right)^{2}-\left(\epsilon_{\alpha p}^{-}\right)^{2}}{p^{\prime}-p}\right. \\
& \times b_{-\alpha-p} b_{-\alpha-p^{\prime}}^{\dagger} e^{-(i t / \hbar)\left(\epsilon_{\alpha p^{\prime}}^{-}-\epsilon_{\alpha p}^{-}\right)}+u_{p} u_{p^{\prime}} \\
& \left.\times \frac{\left(\epsilon_{\alpha p^{\prime}}^{+}\right)^{2}-\left(\epsilon_{\alpha p}^{+}\right)^{2}}{p^{\prime}-p} b_{\alpha p}^{\dagger} b_{\alpha p^{\prime}} e^{-(i t / \hbar)\left(\epsilon_{\alpha p^{\prime}}^{+}-\epsilon_{\alpha p}^{+}\right)}\right\} .
\end{aligned}
$$


In Eq. (9) we have already omitted the terms containing $b_{\alpha p}^{\dagger} b_{-\alpha-p^{\prime}}^{\dagger}$ and $b_{-\alpha-p} b_{\alpha p^{\prime}}$ which vanish after the averaging. The averaged value of the energy current $j_{\epsilon}$ can be written as the sum of two contributions:

$$
j_{\epsilon}=-h^{-1} \sum_{\alpha} \int d p \epsilon_{\alpha p}^{+} \partial_{p} \epsilon_{\alpha p}^{+} v_{p}^{2}+j_{q} .
$$

The first of them can be attributed to the supercurrent flow and cannot transfer heat, whereas $j_{q}$ represents the heat current:

$$
j_{q}=h^{-1} \sum_{\alpha} \int d p \epsilon_{\alpha p}^{+} \partial_{p} \epsilon_{\alpha p}^{+} n\left(\epsilon_{\alpha p}^{+}\right) .
$$

The latter is determined by the energy distributions, $n\left(\epsilon_{\alpha p}^{+}\right)$ and the group velocity, $\partial_{p} \epsilon_{\alpha p}^{+}$of quasiparticles. We express the energy currents (10) and (11) in terms of the "+ "-branch of the spectrum (1) using the relationship $\epsilon_{\alpha p}^{-}=-\epsilon_{-\alpha-p}^{+}$and the symmetry of the limits in the sum. The distribution functions of rightmovers $\left(\partial_{p} \epsilon_{\alpha p 6}^{+}>0\right)$ and leftmovers $\left(\partial_{p} \epsilon_{\alpha p}^{+}\right.$ $<0)$ are assumed to be different and set by reservoirs, as $n\left(\epsilon_{\alpha p}^{+}, T+\Delta T\right)$ and $n\left(\epsilon_{\alpha p}^{+}, T\right)$, respectively. Using this, we determine the thermal conductance $\kappa(T, B)$ given by Eq. (4) as the proportionality coefficient between the heat current and the temperature drop, $j_{q}=\kappa(T, B) \Delta T$.

Fig. 3(a) shows the thermal conductance (4) normalized by that of a normal wire as a function of $k_{B} T / E_{g}$ for different values of the magnetic field. Plot $A$ is related to $B=0$ and shows how the conductance exponentially decreases at temperatures smaller than the minigap $E_{g}$. Curves $B$ and $C$ show what happens when the field crosses the value of $B^{*}$, at which the edge of the minigap is about to reach the Fermi level. For $B<B^{*}$ (curve $B$ ), $\kappa(T) / \kappa_{N}(T)$ is exponentially small only if $k_{B} T<E_{g}-v_{F} \Pi \ll E_{g}$. When the temperature is in the interval $E_{g}-v_{F} \Pi<k_{B} T<E_{g}+v_{F} \Pi$, quasiparticles with negative momenta $p \approx-p_{F}$ transfer heat, whereas the states with positive $p$ are still unpopulated. This interval corresponds to the plato in curve $B$ where the conductance $\kappa(T)$ is half of that in the normal state. At higher temperatures, $k_{B} T>E_{g}+v_{F} \Pi$ the asymmetry of the excitation spectrum no longer matters, and $\kappa(T) \approx \kappa_{N}(T)$.
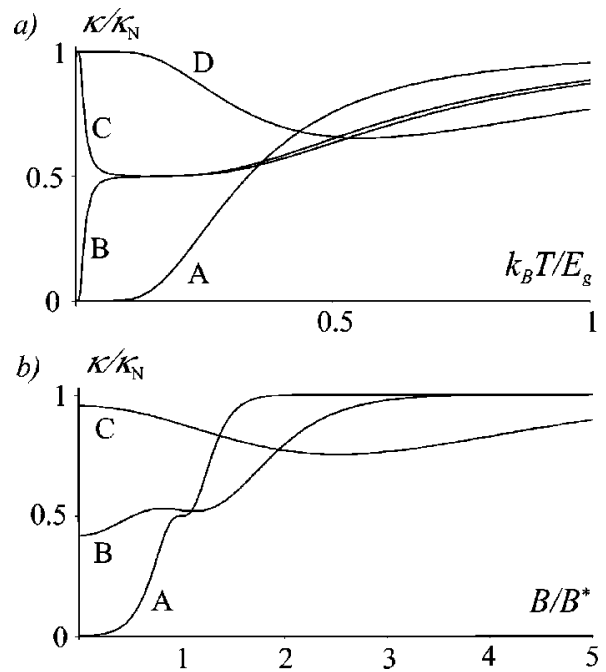

FIG. 3. (a) Temperature dependence of the thermal conductance, $\kappa$ normalized by that of a normal wire, $\kappa_{N}$ for different values of magnetic field: (A) $B / B^{*}=0.01$, (B) $B / B^{*}=0.95$, (C) $B / B^{*}$ $=1.05$, and (D) $B / B^{*}=2$. (b) Magnetic field dependence for different temperatures: (A) $k_{B} T / E_{g}=0.1$, (B) $k_{B} T / E_{g}=0.3$, and (C) $k_{B} T / E_{g}=1$.

When the field exceeds $B^{*}$ (curves $C$ and $D$ ), the dependence $\kappa(T) / \kappa_{N}(T)$ becomes nonmonotonic. As in a normal wire, at low temperatures $k_{B} T \ll v_{F} \Pi-E_{g}$ there are two leftmoving and two right-moving modes capable of tranferring heat, which gives $\kappa(T)=\kappa_{N}(T)$. At intermediate temperatures $v_{F} \Pi-E_{g} \ll k_{B} T \ll E_{g}+v_{F} \Pi$, only the states with negative momenta contribute to the thermal conductance: $\kappa(T)$ $=\kappa_{N}(T) / 2$. At higher temperatures the conductance recovers a normal metallic behavior. Finally, when $B \gg B^{*}$ the minimum in $\kappa(T) / \kappa_{N}(T)$ is less pronounced and the heat conductance behavior becomes indistinguishable from that of a normal wire. The magnetic field dependence of $\kappa / \kappa_{N}$ is given in Fig. 3(b).

The authors thank U. Zulicke, I. Aleiner, and A. Geim for useful discussions. This work was funded in parts by EPSRC (UK) and EC STREP within the Framework 6 EU program.
${ }^{1}$ A.F. Andreev, Zh. Exp. Teor. Fiz. 46, 1823 (1964) [Sov. Phys. JETP 19, 1228 (1964)].

${ }^{2}$ J.B. Ketterson and S.N. Song, Superconductivity (Cambridge University Press, Cambridge, 1998).

${ }^{3}$ B.J. van Wees et al., Phys. Rev. Lett. 69, 510 (1992); S.G. den Hartog et al., ibid. 77, 4954 (1996); A.F. Morpurgo et al., ibid. 78, 2636 (1997); S.G. den Hartog et al., ibid. 79, 3250 (1997); A.F. Morpurgo et al., ibid. 79, 4010 (1997); S.G. den Hartog et al., Phys. Rev. B 56, 13738 (1997).

${ }^{4}$ V.T. Petrashov et al., Phys. Rev. Lett. 70, 347 (1993); 74, 5268 (1995); W. Belzig et al., Phys. Rev. B 66, 220505 (2002).

${ }^{5}$ H. Courtois et al., Phys. Rev. Lett. 76, 130 (1996); P. Dubos et al., ibid. 87, 206801 (2001).

${ }^{6}$ D.A. Dikin et al., Phys. Rev. B 65, 012511 (2002); A. Parsons et al., ibid. 67, 140502 (2003).

${ }^{7}$ A.F. Volkov et al., Physica C 242, 261 (1995); A.F. Volkov, Phys. Lett. A 174, 144 (1993); Pis'ma Zh. Éksp. Teor. Fiz. 55, 713 (1992) [JETP Lett. 55, 746 (1992)].

${ }^{8}$ A. Chrestin et al., Phys. Rev. B 55, 8457 (1997).

${ }^{9}$ C.W.J. Beenakker, Rev. Mod. Phys. 69, 731 (1997); C.J. Lambert and R. Raimondi, J. Phys.: Condens. Matter 10, 901 (1998).

${ }^{10}$ J.B. Pendry, J. Phys. A 16, 2161 (1983).

${ }^{11}$ U. Zulicke et al., Physica B 298, 453 (2001). 